Recebido em 11/2019. Aceito para publicação em 01/2020.

\title{
APLICAÇÃO DE ESPECTROSCOPIA NO INFRAVERMELHO: COMO FERRAMENTA PARA ANÁLISE QUANTITATIVA DE ORÉGANO
}

\section{APPLICATION OF INFRARED SPECTROSCOPY: AS A TOOL FOR QUANTITATIVE ANALYSIS OF OREGANO}

\author{
Vitória Fontes ${ }^{1}$ \\ Douglas Cubas Pereira ${ }^{2}$ \\ Breno Pupin ${ }^{3}$ \\ Kumiko Koibuchi Sakane ${ }^{4}$
}

Resumo: Desde a antiguidade as ervas aromáticas são utilizadas, não somente para melhorar o sabor e odor em alimentos, mas também para estender o tempo de prateleira com suas propriedades antissépticas. O aumento da utilização das ervas aromáticas na culinária tem sido aprimorado praticamente no mundo todo e principalmente na gastronomia. Entre as ervas mais consumidas no Brasil, o orégano (Origanum vulgare) tem origem mediterrânea e trata-se de uma erva muito aromática, com notas de cânfora, sabor ardido e picante. O presente estudo tem como objetivo analisar quantitativamente e qualitativamente a composição biomolecular das amostras de orégano utilizando técnicas espectroscópicas no infravermelho com transformada de Fourier (FT-IR). Com os resultados, foi possível notar diferenciação na quantidade de lignina, água e lipídio, e a análise multivariada permitiu identificar a similaridade das amostras analisadas. O uso de espectroscopia no infravermelho tem se mostrado uma ótima ferramenta na análise de alimentos, podendo ser aplicada em conjunto com outras técnicas na indústria de alimentos.

Palavras-chave: Espectroscopia no infravermelho; FTIR; orégano; análise multivariada.

Abstract: Since ancient times, aromatic herbs have been used not only to improve taste and odor in foods but also to extend shelf life with their antiseptic properties. The increased use of aromatic herbs in cooking has been improved practically worldwide and especially in gastronomy. Among the most consumed herbs in Brazil, oregano (Origanum vulgare) has a Mediterranean origin and is a very aromatic herb, with notes of camphor, burning and spicy flavor. The present study aims to quantitatively and qualitatively analyze the biomolecular composition of oregano samples using Fourier Transform Infrared (FT-IR) spectroscopic techniques. With the results, it was possible to notice differentiation in the amount of lignin, water and lipid, and the multivariate analysis allowed the identification of the similarity of the analyzed samples. The use of infrared spectroscopy has proven to be a great tool in food analysis and can be applied together with other techniques in the food industry.

Keywords: Infrared spectroscopy; FTIR; oregano; multivariate analysis.

\section{INTRODUÇÃO}

Desde a antiguidade, as ervas aromáticas são utilizadas e acompanharam as migrações e a evolução dos povos que as utilizavam, inclusive protegendo a saúde, devido às suas propriedades antimicrobianas que evitam algumas infecções (TEJADA

\footnotetext{
1 Mestranda em Processamento de Materiais. Universidade do Vale do Paraíba - Univap, Brasil. E-mail: fontes.vitoria@gmail.com.

2 Mestranda em Processamento de Materiais. Universidade do Vale do Paraíba - Univap, Brasil. E-mail: douglas.cubas@hotmail.com.

${ }^{3}$ Docente na Universidade do Vale do Paraíba - Univap, Brasil. E-mail: breno0891@hotmail.com.

4 Docente na Universidade do Vale do Paraíba - Univap, Brasil. E-mail: kumiko@univap.br.
} 
et al., 2016). Estudos mostram relatos de historiadores como Hipócrates (pai da medicina) e outros que fizeram descobertas do uso das ervas aromáticas com a finalidade de tratar doenças atrelados aos princípios filosóficos (PINTO, 2014).

As especiarias e ervas aromáticas têm sido usadas não somente para melhorar o sabor e odor em alimentos e estender o tempo de prateleira, mas também pelas suas propriedades antissépticas e medicinais (NIETO, 2017), além disso apresentam propriedades biológicas, como óleos essenciais, também conhecidos como substâncias ativas, por exemplo, os compostos fenólicos que constituem os óleos essenciais, assim como os taninos, álcoois, ésteres, aldeídos, entre outros elementos (HUSSEIN; ELANSSARY, 2018).

Antes de surgir a escrita, já se fazia o uso de ervas aromáticas para fins alimentares nas civilizações antigas, que por meio de experimentos, os povos utilizavam as ervas e foram descobrindo suas combinações e seus sabores picantes e adocicados (STOBART, 2017). Na Europa, após tantas descobertas a partir do uso medicinal, surgiu o interesse de usá-las para fins alimentícios onde incrementariam o sabor dos alimentos. Logo, na descoberta do Brasil os portugueses trazem seus conhecimentos já adquiridos sobre ervas aromáticas e encontram os índios que usavam urucum para pintar, para se protegerem de insetos e tingir seus objetos (CHEVALLIER, 2016).

As ervas aromáticas ou ervas-de-cheiro são plantas, geralmente de pequenas dimensões, que apresentam diversas utilizações e propriedades (VAN WYK; WINK, 2018). Segundo Kapadiya et al (2016), a folha de uma planta utilizada na culinária pode ser chamada de erva culinária, e qualquer outra parte da planta, geralmente parte seca, é chamada de especiarias. As especiarias podem ser os gomos (cravo), cascas (canela), raízes (gengibre), frutos (pimenta), sementes aromáticas (cominho), e mesmo o estigma da flor (açafrão). Para exemplificar, tem-se o exemplo do coentro, cujas folhas são referidas como uma erva e as sementes secas como uma especiaria (KABAK; DOBSON, 2017).

O aumento da utilização das ervas aromáticas na culinária tem sido aprimorado praticamente no mundo todo, principalmente na gastronomia, pois realça o sabor e evidencia o aroma do alimento (THOMAS, 2016), dentre as ervas aromáticas mais utilizadas está o orégano (Origanum vulgare) (MORSHEDLOO et al., 2017), que provém do grego "oros" e "ganos" e que significa "adorno ou alegria da montanha", devido ao seu aspecto e aroma agradável. Utilizados desde a Roma e Grécia antiga, foi levado à Inglaterra pelos romanos, com notas de cânfora sabor ardido e picante (CHEVALLIER, 2016), já, com relação à sua origem mediterrânea, possui 38 espécies catalogadas, com folhas pequenas de coloração verde escura. Na culinária brasileira é mais utilizado no preparo de carnes, ovos, peixes, panificação e frutos do mar (DALBY; DALBY, 2017), além disso, o óleo essencial de orégano também é utilizado na perfumaria (VIVAS et al., 2017), e na indústria alimentícia visando controlar microrganismos causadores de deterioração e/ou causadores de doenças veiculadas por alimentos (FORSYTHE, 
2020).

É um condimento muito consumido no mercado brasileiro e pouco produzido nacionalmente, fator que leva o Brasil a importar de outros países como o Chile e países do Mediterrâneo (FORSYTHE, 2020). Assim as pesquisas têm sido intensificadas nos últimos anos visando maximizar o controle de qualidade do processo produtivo de orégano no Brasil e reduzir as quantidades importadas (DALBY \& DALBY, 2017).

Hoje, observa-se uma evolução no que se diz respeito aos padrões de qualidade e pureza dos alimentos, sobretudo a respeito da redução do emprego de produtos sintéticos desde a sua produção, por exemplo de agrotóxicos, antioxidantes sintéticos e corantes.(BOPP, 2019), Neste contexto, novas técnicas na identificação de pureza dos alimentos têm sido aplicadas, a exemplo da espectroscopia no infravermelho que permite identificar características estruturais das moléculas, tornando-se claro que mesmo uma molécula muito simples pode dar um espectro muito complexo (DAZZI; PRATER, 2017).

A espectroscopia no infravermelho médio com Transformada de Fourier (FTIRUATR) é uma técnica analítica moderna de larga aplicação em diversas áreas da pesquisa científica, devido à sua simplicidade do preparo de amostras, por não produzir resíduos e ser extremamente rápida e eficiente (DEPCIUCH et al., 2017).

Uma das grandes vantagens da espectroscopia no infravermelho é que é possivel estudar qualquer estado físico, permite análises rápidas, limpas e sem necessidade de reagentes agressivos e tratamento da amostra (DEPCIUCH et al., 2016). A espectroscopia no infravermelho médio vem se desenvolvendo nos últimos anos e pode ser uma excelente ferramenta para análise quantitativa e qualitativa da amostra (DEBRUYNE; DELANGHE; SPEECKAERT, 2018).

\section{OBJETIVO}

O presente estudo tem como objetivo analisar quantitativamente $\mathrm{e}$ qualitativamente a composição biomolecular das amostras de orégano, utilizando técnicas espectroscópicas no infravermelho com transformada de Fourier (FT-IR). Como objetivo específico, são comparadas as bandas características das principais biomoléculas constituintes do orégano por meio da análise estatística multivariada.

\section{METODOLOGIA}

As amostras de orégano (figura 1) são produtos industrializados de origem brasileira, de produtores diferentes, e comercializados no mercado. Foram compradas no dia 27 de maio de 2019 em dois mercados de São José dos Campos. 
Figura 1 - Amostras dos oréganos analisados.

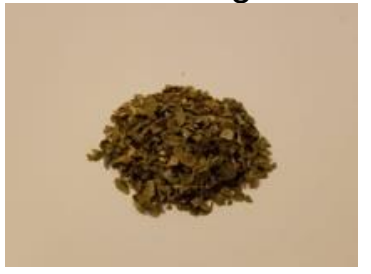

A

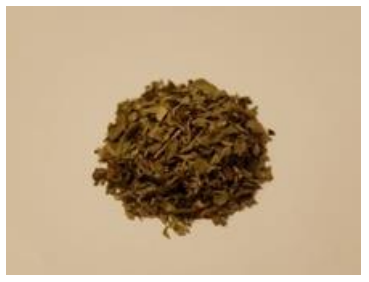

D

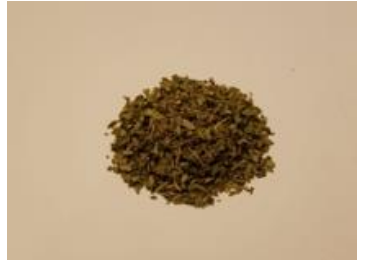

B

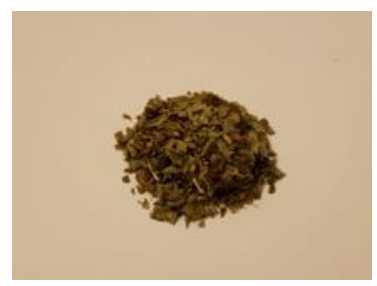

$\mathbf{E}$

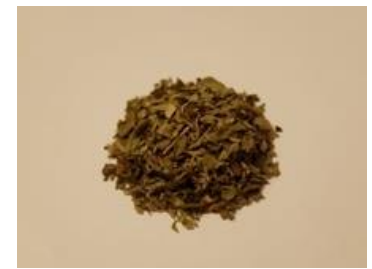

C

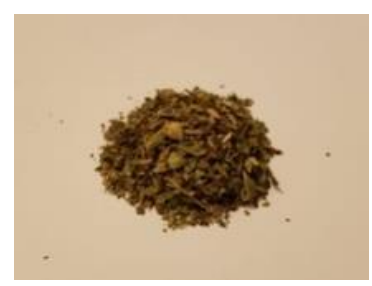

$\mathbf{F}$

Fonte: Os autores.

As amostras foram divididas em seis grupos, de acordo com a quantidade de fabricantes (marcas), a análise das amostras foi feita em triplicata e obtenção de dados foi realizado no Laboratório de Espectroscopia Vibracional no Infravermelho do Instituto de Pesquisa e Desenvolvimento (IP\&D) da Universidade Vale do Paraíba (UNIVAP), em São José dos Campos.

As amostras selecionadas foram maceradas no almofariz de ágata, com o auxílio do pistilo de ágata até que atingissem a forma de pó, e inseridas na porta amostra do espectrofotômero para a obtenção dos espectros infravermelhos.

A região escolhida foi na faixa de 4000 a $450 \mathrm{~cm}^{-1}$ (região do infravermelho médio - MIR) com resolução de $4 \mathrm{~cm}^{-1}$ a temperatura ambiente. O espectrofotômero utilizado foi Spectrum Two (figura 2), com transformada de Fourier de PekinElmer.

Os espectros infravermelhos foram processados com software Spectrum 5.3 (PerkinElmer) e foram feitas correções de linha de base, suavização espectral utilizando o algarismo Savistzky Golay (9 pontos), normalização e colocação em absorbância. Após a formatação foi obtido o espectro médio a partir dos três espectros selecionados. 
Figura 2 - Spectrum Two (FTIR-UATR)

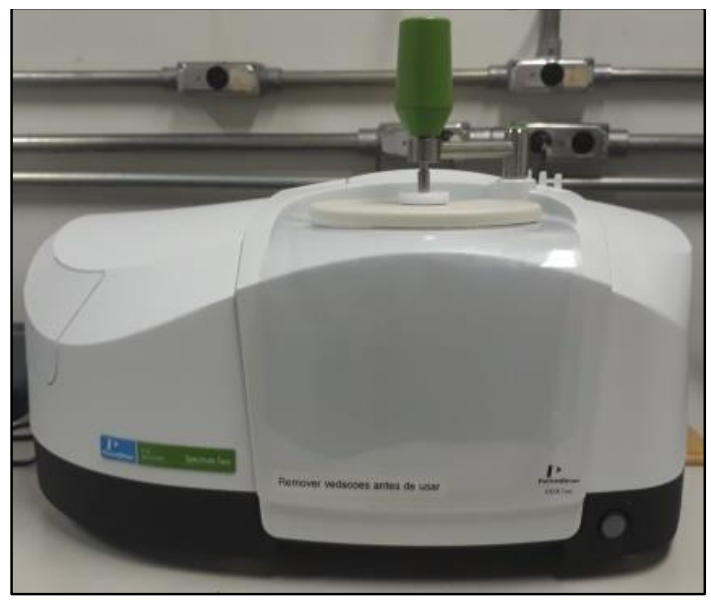

Fonte: Os autores.

Com o auxílio do software Minitab 17, os espectros foram submetidos à análise multivariada para verificar a similariadade nas diferentes amostras.

\section{RESULTADOS}

Os espectros das amostras de orégano foram comparados entre si, com a finalidade de identificar diferenças nos contornos de banda que indicam possíveis diferenças de cada produtor (marca), conforme pode ser visto na figura 3.

A Tabela 1 apresenta as atribuições aproximadas das bandas de absorção das amostras de orégano.

Para verificar a similaridade das amostras de orégano, os espectros foram submetidos à análise multivariada, o dendrograma (figura 4) obtido apresentou seis grupos, sendo A - (1, 2 e 3); B - (4, 5 e 6); C - (7, 8 e 9); D - (10, 11 e 12); E - (13, 14 e $15) ; \mathrm{F}-(16,17$ e 18). Consideram-se os autovalores (eigenvalues) até PC3 (figura 5) que apresentam maior significância por meio de scree test (SRIVASTAVA et al., 2018). 
Figura 3 - Espectros infravermelhos das amostras na faixa de 4000 a $450 \mathrm{~cm}^{-1}$.

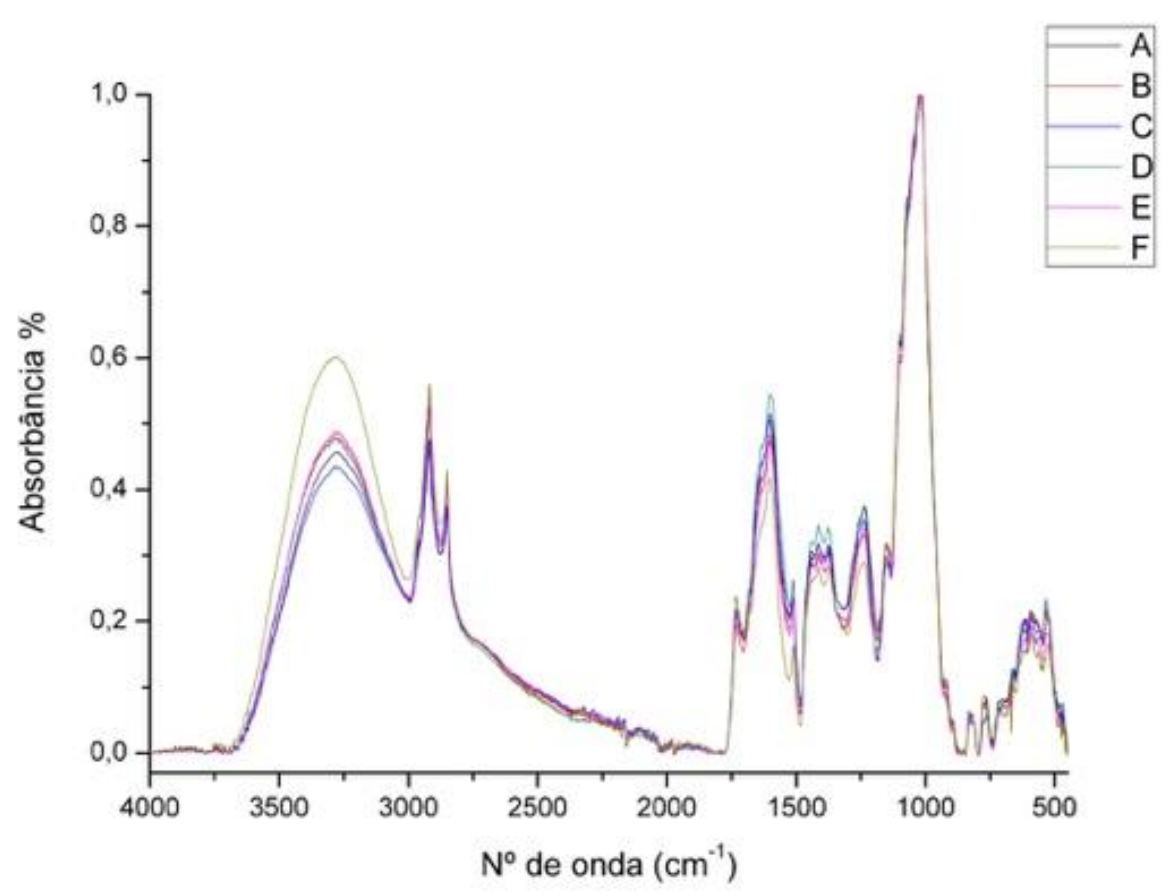

Fonte: Os autores.

Tabela 1 - Modos vibracionais e suas atribuições aproximadas.

\begin{tabular}{|c|c|}
\hline $\begin{array}{l}\text { Número de } \\
\text { onda }\left(\mathrm{cm}^{-1}\right)\end{array}$ & Modo vibracional e atribuição aproximada \\
\hline$\sim 3610$ & Estiramento da ligação O-H. \\
\hline$\sim 2930$ & $\begin{array}{l}\text { Estiramento assimétrico do } \mathrm{CH} \text { nos grupos } \mathrm{CH}_{2} \text { ou } \mathrm{CH}_{3} \text {, do } \\
\text { lipídio. }\end{array}$ \\
\hline$\sim 2850$ & Estiramento simétrico $\mathrm{CH}_{2}$, do lipídio. \\
\hline$\sim 1750$ & Estiramento $\mathrm{C}=\mathrm{O}$ do grupo carbonila, pectina. \\
\hline$\sim 1610$ & Estiramentode anel aromático, lignina. \\
\hline$\sim 1500$ & $\begin{array}{l}\text { Deformação } \mathrm{NH} \text { e estiramento } \mathrm{CN} \text { do grupo peptídico em } \\
\text { proteína, amida secundária. }\end{array}$ \\
\hline$\sim 1320$ & Estiramento simétrico do $\mathrm{COO}^{-}$, clorofila. \\
\hline$\sim 1000$ & Estiramento C-O-C e C-O-H. \\
\hline$\sim 760$ & Deformação no plano, wag, de $\mathrm{OH}$ de catequina. \\
\hline
\end{tabular}

Fonte: Depciuch et al. (2017); Selvaraj et al. (2018); Rajkumar et al., (2018); Teixeira et al., (2018); Minteguiaga et al., (2019). 
Figura 4 - Análise multivariada dos espectros das seis amostras de orégano.

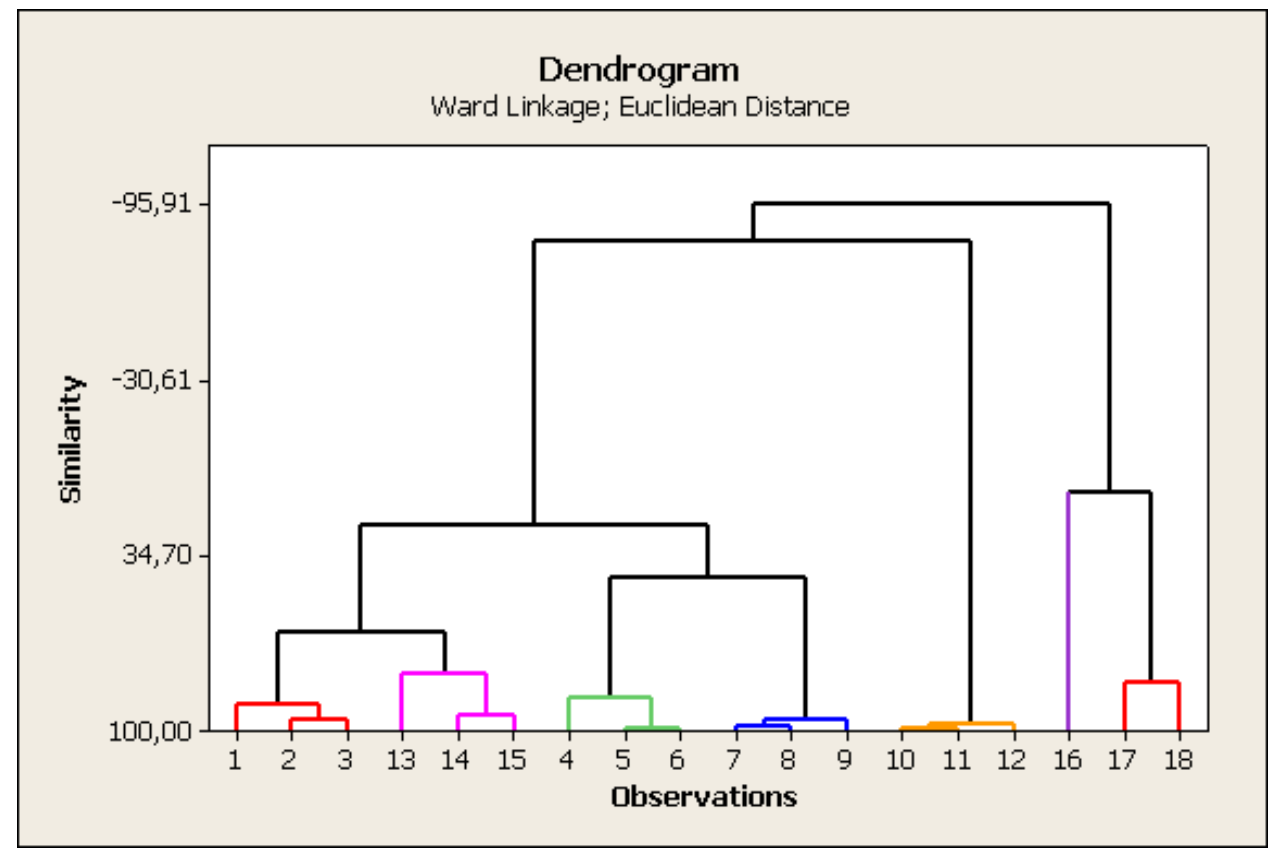

Fonte: Os autores.

Figura 5 - Scree Plot dos espectros avaliados

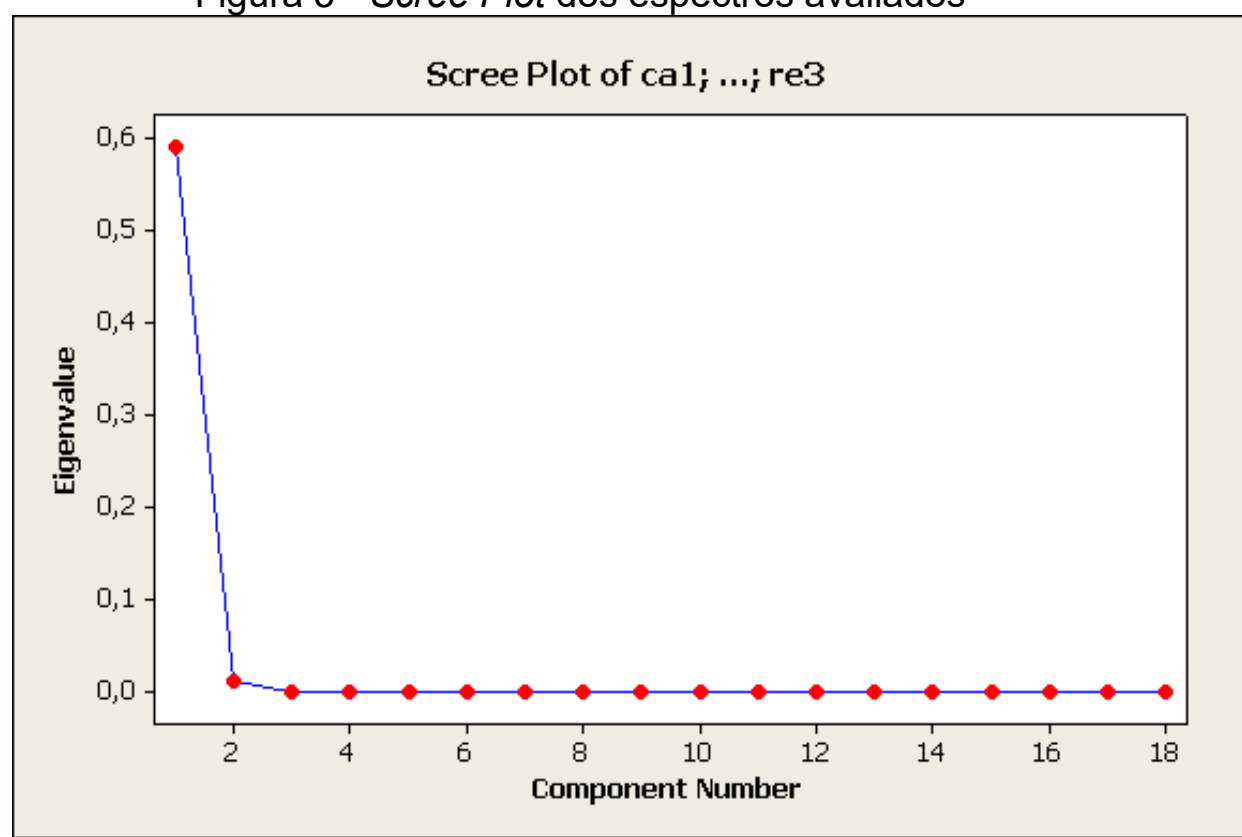

Fonte: Os autores.

\section{DISCUSSÃO}

O resultado da análise multivariada (figura 4) demonstra que todos os grupos se separaram em grupos homogêneos: os grupos A (1, 2 e 3), B (4, 5 e 6), C (7, 8 e 9), D (10, 11 e 12), E (13, 14 e 15), apresentaram similaridade significativa, 90,51\%, 78,69\%, 
$88,20 \%, 96,06 \%, 97,50 \%$, respectivamente. A amostra $F(16,17$ e 18$)$ apresentou baixo índice de homogeneidade com $11,54 \%$.

Sugere-se que a similaridade de cada amostra é influenciada pela quantidade de cada embalagem que é comercializada pelo fabricante, amostra A (15g), amostra B $(30 \mathrm{~g})$, amostra C $(10 \mathrm{~g})$, amostra D $(8 \mathrm{~g})$, amostra $E(38 \mathrm{~g})$, amostra $F(500 \mathrm{~g})$, considerando que a amostra $\mathrm{F}$ possui maior amostragem, possivelmente maior índice de heterogeneidade. Além disso, os fatores que influenciam plantas são diversos, os internos como características genéticas e os externos como a presença de nutrientes no solo e diferentes níveis de radiação solar (BELYUCHENKO, 2016).

A banda com número de onda de $1610 \mathrm{~cm}^{-1}$ indica estiramento de anel aromático (DEPCIUCH et al., 2017) que se relaciona à presença de lignina, uma molécula fenólica altamente complexa em plantas, com função de conferir rigidez, impermeabilidade e resistência ao tecido vegetal, variando de 20 a $40 \%$ da massa seca das plantas (RENCORET et al., 2018).

A amostra $D$ apresentou maior quantidade de lignina e a amostra $F$ com menor quantidade de lignina. Considerando que a lignina confere impermeabilidade à planta, à banda de onda $3610 \mathrm{~cm}^{-1}$ que está relacionada a presença de água na amostra, observa-se que nesses termos, a quantidade de água livre que não se encontra comprometida com as moléculas constituintes do produto, está disponível para as reações físicas, químicas e biológicas (CHEMAT et al., 2017). Sendo responsável pela deterioração em alimentos, neste caso, a amostra $\mathrm{F}$ apresentou o maior índice de água na amostra, fator que se explica pela quantidade da amostragem, que possivelmente fica mais exposto ao ar e às contaminações externas (manejo e mistura com outras ervas aromáticas e condimentos). Na literatura, é utilizado tanto o termo umidade ou atividade de água, para se referir à quantidade de água presente no alimento, sendo frequente pensar que a maior estabilidade do alimento está no controle de umidade mínima (CHEMAT et al., 2017).

A banda com número de onda $1320 \mathrm{~cm}^{-1}$ indica a presença de clorofila (pigmento verde das plantas). A amostra $D$ apresentou maior quantidade de clorofila visualmente. A amostra tem a coloração verde mais aparente comparado as outras amostras analisadas.

Segundo Divekar et al (2017), os lipídios estão na região de espectro entre 2800 a $3000 \mathrm{~cm}^{-1}$. Em alimentos, determina o sabor, a textura e aparência, conferindo valor nutritivo e energia. As amostras F e D apresentaram maior índice de lipídio, pois, de acordo com Oliva et al (2016), os lipídios fixam e ressaltam o sabor dos alimentos, sugerindo, assim que provavelmente são mais saborosos, influenciando na preparação e sabor final do alimento.

$\mathrm{Na}$ gastronomia, quando o homem passou a utilizar temperos para aumentar o tempo do alimento, diminuindo a sua deterioração, iniciou a potencialidade das ervas 
aromáticas, as quais também incrementavam o paladar dos alimentos. Anos depois, houve grandes transformações sóciopolíticas, como o fim do Império Romano e o início dos descobrimentos, além da sua importância histórica, o avanço nos métodos de análise em alimentos nos permite identificar nutrientes e otimizá-los com diferentes tipos de cocção.

\section{CONCLUSÃO}

O uso de espectroscopia no infravermelho tem se mostrado uma ótima ferramenta na análise de alimentos, devido à facilidade e rapidez do uso da técnica, além disso não necessita de tratamento da amostra, fator que na análise de alimentos pode causar diferenças na estrutura molecular. É uma técnica de baixo custo e avançada, podendo ser aplicada em conjunto com outras técnicas na indústria de alimentos.

\section{AGRADECIMENTOS}

Os autores agradecem o apoio financeiro pela FVE.

\section{REFERÊNCIAS}

BELYUCHENKO, I. S. Cultivated Lands of Kuban and Features of Their Development. International Journal of Environmental and Science Education, $v$. 11, n. 13, p. 6255-6276, 2016.

BOPP, A. F. The Evolution of Food Preservation and Packaging. In: Chemistry's Role in Food Production and Sustainability: Past and Present. American Chemical Society, p. 211-228, 2019.

CHEMAT, F. et al. Review of green food processing techniques. Preservation, transformation, and extraction.Innovative Food Science \& Emerging Technologies, v. 41, p. 357-377, 2017.

CHEVALLIER, A. Encyclopedia of Herbal Medicine: 550 Herbs and Remedies for Common Ailments. Penguin, 2016.

DALBY, A.; DALBY, R. Gifts of the Gods: a history of food in Greece. [s.I.]: Reaktion Books, 2017.

DAZZI, A.; PRATER, C. B. AFM-IR: technology and applications in nanoscale infrared spectroscopy and chemical imaging. Chemical reviews., v. 117, n. 7, p. 5146-5173, 2017.

DEBRUYNE, S.; DELANGHE. J. R.; SPEECKAERT, M. M. Applications of mid-infrared spectroscopy in the clinical laboratory setting. Critical reviews in clinical laboratory sciences., v. 55, n. 1, p. 1-20, 2018.

DEPCIUCH, J. et al. FTIR analysis of molecular composition changes in hazel pollen from unpolluted and urbanized areas. Aerobiologia, v. 33, n. 1, p. 1-12, 2017. 
DEPCIUCH, J. et al. Application of Raman spectroscopy and infrared spectroscopy in the identification of breast cancer. Applied spectroscopy, v. 70, n. 2, p. 251-263, 2016.

DIVEKAR, M. T. et al. Effect of microwave treatment on the cooking and macronutrient qualities of pulses. International Journal of Food Properties, v. 20, n. 2, p. 409-422, 2017.

FORSYTHE, S. J. The microbiology of safe food. [s.I.]: John Wiley \& Sons, 2020.

HUSSEIN, R. A.; EL-ANSSARY, A. A. Plants secondary metabolites: the key drivers of the pharmacological actions of medicinal plants. In: BUILDERS, P. F. Herbal Medicine. [s.]: Intechopen, 2018.

KABAK, B.; DOBSON, A. D. W. Mycotoxins in spices and herbs-An update. Critical reviews in food science and nutrition, v. 57, n. 1, p. 18-34, 2017.

KAPADIYA, D. B. et al. Spices and herbs as a source of natural antioxidants for food. International Journal of Current Microbiology and Applied Sciences, v. 5, n. 7, p. 280-288, 2016.

MINTEGUIAGA, M. et al. FT-IR, FT-Raman, UV-Vis, NMR and structural studies of carquejyl acetate, a distinctive component of the essential oil from Baccharis trimera (less.) DC.(Asteraceae). Journal of Molecular Structure, v. 1177, p. 499-510, 2019.

MORSHEDLOO, M. R. et al. Effect of prolonged water stress on essential oil content, compositions and gene expression patterns of mono-and sesquiterpene synthesis in two oregano (Origanum vulgare L.) subspecies. Plant physiology and biochemistry, v. 111, p. 119-128, 2017.

NIETO, G. Biological activities of three essential oils of the Lamiaceae family. Medicines, v. 4, n. 3, p. 63, 2017.

OLIVA, L. et al. In rats fed high-energy diets, taste, rather than fat content, is the key factor increasing food intake: a comparison of a cafeteria and a lipid-supplemented standard diet. PeerJ, v. 5, p. e3697, 2017.

PINTO, E. O. A. "Ervas aromáticas”, suas contribuições para uma alimentação saudável. Universidade Tecnológica Federal do Paraná. Monografia de especialização, p. 12-13, 2014.

RAJKUMAR, P. et al. Vibrational and electronic spectral analysis of thymol an isomer of carvacrol isolated from Trachyspermum ammi seed: A combined experimental and theoretical study. Chemical Data Collections, v. 15, p. 10-31, 2018.

RENCORET, J. et al. Variability in lignin composition and structure in cell walls of different parts of Macaúba (Acrocomia aculeata) palm fruit. Journal of agricultural and food chemistry, v. 66, n. 1, p. 138-153, 2018.

SELVARAJ, S. et al. Vibrational (FT-IR and FT-Raman), electronic (UV-vis) and quantum chemical investigations on pyrogallol: A study on benzenetriol dimers. Vibrational Spectroscopy, v. 95, p. 16-22, 2018.

SRIVASTAVA, S. et al. Identification and differentiation of insect infested rice grains varieties with FTNIR spectroscopy and hierarchical cluster analysis. Food chemistry, 
v. 268, p. $402-410,2018$.

STOBART, T. Herbs, Spices \& Flavourings. [s.]]: Overlook Books, 2017.

TEIXEIRA, A. M. R. et al. Vibrational spectroscopy study of essential oils from Plectranthus amboinicus Lour. Spreng and Vanillosmopsis arborea Baker. Vibrational Spectroscopy, v. 98, p. 22-29, 2018.

TEJADA, S. et al. Wound healing effects of curcumin: A short review. Current pharmaceutical biotechnology, v. 17, n. 11, p. 1002-1007, 2016.

VAN WYK, B. E.; WINK, M. Medicinal plants of the world. [s.I]: CABI, 2018.

VIVAS, F. E. et al. Elaboration of essential oil from the oregano for medicinal use sheet. International journal of physical sciences and engineering, v. 1, n. 1, p. 8187, 2017. 